\title{
Management von schadstoffbelasteten Feinsedimenten in Bundeswasserstraßen
}

\author{
Vera Breitung • Martin Keller
}

Eingegangen: 19. August 2010/Akzeptiert: 13. September 2010/Online veröffentlicht: 14. Oktober 2010

(C) Springer-Verlag 2010

Zusammenfassung Schon vor mehr als zehn Jahren wurden unter der Federführung der BfG Handlungsanweisungen für den Umgang mit Baggergut im Geltungsbereich der Wasser- und Schifffahrtsverwaltung erstellt, wobei die zu betrachtenden Sedimente vorrangig hinsichtlich ihrer Qualität zu beurteilen sind. Die möglichen Auswirkungen auf die Umwelt an der Entstehungs- und Umlagerungsstelle sind dabei immer unter ökologischen und ökonomischen Gesichtspunkten zu bewerten.

Neuere Entwicklungen auf diesem Gebiet stellt der kürzlich verabschiedete Sedimentmanagementplan am Rhein dar, der eine Erfassung von Menge und Qualität von mit Schadstoff belastetem Sediment, eine Bewertung und Klassifizierung der Sedimente gemäß ihres Gefährdungspotenzials sowie Vorschläge zum ökologischen und ökonomischen Umgang beinhaltet und die derzeit gültigen Handlungsanweisungen entsprechend berücksichtigt.

Eine Weiterentwicklung der Handlungsanweisungen zum Umgang mit Baggergut und Sedimenten in Bundeswasserstraßen aufgrund der geänderten fachlichen und rechtlichen Situation ist angestrebt. Hierbei müssen die Ziele der Flussgebietsgemeinschaften und der Nutzer der Gewässer beiderseits angemessen berücksichtigt werden.

Im Rahmen von Untersuchungen des möglichen Einflusses der klimatischen Veränderungen auf das Sedimentmanagement werden ein- und mehrdimensionale Rechenmodelle zum Einsatz kommen, die neben einer erweiterten

Verantwortliche Herausgeber: Organisationskomitee der Tagung

V. Breitung $(\bowtie) \cdot$ M. Keller

Bundesanstalt für Gewässerkunde,

Mainzer Tor 1, 56168 Koblenz, Deutschland

E-Mail: breitung@bafg.de
Prozesskenntnis auch Prognosen über die Veränderung des Schadstofftransportes im Gewässer liefern sollen.

Schlüsselwörter Bundeswasserstraßen .

Handlungsstrategien · Sedimentbewertung ·

Sedimentmanagement $\cdot$ Umlagerung

\section{Management of contaminated fine grained sediments in federal water ways}

Abstract The Federal Institute of Hydrology has developed operating strategies in order to deal with dredged material within the scope of the Waterways and Shipping Administration. Sediments will be assessed based on certain quality parameters. The potential environmental impact at the source and relocation areas has to be evaluated in respect to ecological and economical issues.

A coordinated sediment management plan for the Rhine River was commissioned. The most important contaminated sedimentation areas were identified, and special sedimentation areas were classified based on ecological impact or even risk. Proposals for economical and ecological strategies are discussed.

Further developments for operational instructions dealing with sediments and dredged material in federal waterways are still being processed. The objectives of the river basin commissions as well as those of the stakeholders have to be met in an appropriate manner.

In the framework of the analysis and impact of climate change on both sediment quantity and quality for optimizing sediment management, one or more dimensional hydraulic models will be applied. This will help gain a better insight into the understanding of contaminant transport in freshwater bodies. 
Keywords Federal navigable waterways · Operation strategies $\cdot$ Relocation $\cdot$ Sediment assessment $\cdot$ Sediment management

\section{Einleitung}

Die Nutzung eines Flusses als Wasserstraße erfordert regelmäßig Unterhaltungsmaßnahmen zur Beseitigung von Grob- und Feinsedimenten. Muss aufgrund von Ablagerungen in den Fahrrinnen und Häfen durch natürlich auftretende Sedimentation von Schwebstoffen und Geschiebematerial dort anfallendes Sediment entfernt werden, entscheidet die Qualität des Sedimentes über die Art der Verbringung. Die Verwendungs-, Verwertungs- und Beseitigungsmöglichkeiten von Baggergut an Land und in Gewässern hängen von der physikalischen und chemischen Beschaffenheit und den technischen, ökonomischen und infrastrukturellen Randbedingungen ab. Baggergut ist somit hinsichtlich des Schadstoffpotenzials und der physikalischen Beschaffenheit zu beurteilen, wobei die Beurteilung zu sowohl ökologisch als auch ökonomisch vertretbaren Lösungen führen soll. Die rechtlichen Grundlagen für die Unterhaltungstätigkeiten der Wasser- und Schifffahrtsverwaltung (WSV) an Bundeswasserstraßen bilden die $\S \S 7$ und 8 des Wasserstraßengesetzes (WaStrG). Danach ist die Unterhaltung Hoheitsaufgabe des Bundes und umfasst u. a. die Gewährleistung der Leichtigkeit und Sicherheit der Schifffahrt und die Erhaltung eines ordnungsgemäßen Zustandes des Wasserabflusses.

\section{Regelungen}

Zur Beurteilung der Sedimente sind je nach Unterbringungsweg umfangreiche Vorschriften aus den unterschiedlichsten Rechtsbereichen zu beachten. Die allgemeinen und die stofflichen Qualitätsanforderungen u. a. folgender Rechtsgebiete sind bei Bewertungen zu berücksichtigen:

- Wasserrecht (Wasserhaushaltsgesetz WHG),

- Naturschutzrecht (Bundesnaturschutzgesetz BNatSchG, FFH-Richtlinie),

- Abfallrecht (Kreislaufwirtschafts- und Abfallgesetz $\mathrm{KrW}-/ \mathrm{AbfG}$, Deponieverordnung DepV) und

- Bodenschutzrecht (Bundesbodenschutzgesetz BBodSchG, Bundesbodenschutzverordnung BBodSchV).

Darüber hinaus existieren spezifische Regeln für Baggergut in einzelnen Bundesländern. Die am 22.12.2000 in Kraft getretene Europäische Wasserrahmenrichtlinie (WRRL) gilt als Meilenstein für das Umdenken in der Wasserwirtschaft, da hier der integrierte Gewässerschutz im Mittelpunkt der wasserwirtschaftlichen Planung und Entscheidungen steht. Obwohl nicht explizit in der Richtlinie erwähnt, tragen die
Sedimente im Gewässer in erheblichem Maße zur Qualität des Wasserkörpers bei. Die bis Ende 2009 unter Berücksichtigung der bereits erfolgten Bestandsaufnahmen in den einzelnen Flussgebietseinheiten zu erstellenden und ab $2015 \mathrm{zu}$ überprüfenden Maßnahmenprogramme und Bewirtschaftungspläne sollten somit auch Baggergut- und Sedimentmanagementpläne enthalten, um den guten ökologischen und chemischen Zustand der Wasserkörper langfristig zu sichern.

\section{Aktuelle Handlungsstrategie innerhalb der WSV}

\subsection{Umlagerung}

Werden Sedimente in den Bundeswasserstraßen umgelagert, sind die Handlungsanweisungen des Bundes bei dem Umgang mit Baggergut im Binnenbereich (HABAB-WSV) bzw. im Küstengebiet (Gemeinsame Übergangsbestimmungen, BMVBS) und, so weit vorliegend, auch Empfehlungen der internationalen Flussgebietskommissionen zu beachten. Die genannten Handlungsanweisungen orientieren sich im Allgemeinen am sogenannten Vorsorgeprinzip, d.h. die Gewässerqualität soll durch eine Baggergutverbringung nicht verschlechtert werden. Die Beurteilung soll zu ökonomisch und ökologisch vertretbaren Lösungen führen. Aus diesem Grund hat das Verkehrsministerium (BMVBS) unter Federführung der Bundesanstalt für Gewässerkunde (BfG) die HABAB eingeführt. Untersuchungs- und Bewertungsgegenstand sind die Beschaffenheit der zu verbringenden Sedimente und die möglichen Auswirkungen auf die Umwelt an der Unterbringungsstelle. Der Untersuchungsumfang erstreckt sich auf:

- sedimentologische Untersuchungen (z.B. Korngrößenverteilung),

- hydromorphologische Untersuchungen (Transport, Trübung),

- chemische und biochemische Untersuchungen (chemische Verunreinigungen, Sauerstoffzehrung und Nährstoffe),

- ökotoxikologische Untersuchungen (Biotests) und

- faunistische Untersuchungen (Artenverteilung und -anzahl).

Eine Umlagerung in Binnengewässer wird ausgeschlossen, wenn eine nachhaltige Störung des Wasser- und Feststoffhaushaltes, eine gravierende Änderung des Fließquerschnittes, eine Überschreitung der Schadstoffkonzentration (bezogen auf die Feinkornfraktion $<20 \mu \mathrm{m}$ ) um mehr als das Dreifache der entsprechenden Schadstoffkonzentration im Schwebstoff an der Umlagerungsstelle auftritt oder Beeinträchtigungen hinsichtlich toxischer Effekte zu befürchten sind.

Für die Beurteilung der chemischen Verunreinigungen im Sediment sind folgende Fälle zu unterscheiden: 
- Überschreitet die mittlere Konzentration jedes einzelnen Schadstoffs im Baggergut den entsprechenden Vergleichswert um höchstens das 1,5-fache (Fall 1), so kann umgelagert werden.

- Wird dieser Vergleichswert durch eine Kenngröße um das 1,5- bis 3-fache überschritten (Fall 2), so ist eine Einzelfallbetrachtung anzustellen. In diese Einzelfallbetrachtung sind vor allem Aussagen über das Freisetzungspotenzial aus Elutionstests und Biotestergebnisse einzubeziehen. Darüber hinaus sollten Frachtbetrachtungen angestellt sowie die Sauerstoffverhältnisse und hydrologische und jahreszeitliche Aspekte berücksichtigt werden.

- Über das 3-fache hinausgehende Überschreitungen bei Konzentrationen von organischen Schadstoffen oder Schwermetallen (Fall 3) verbieten prinzipiell eine Umlagerung.

Zur Charakterisierung der von einer Umweltprobe auf einen Modellorganismus ausgehenden Toxizität dient gemäß HABAB-WSV der pT-Wert (potentia toxicologiae $=$ toxikologischer Exponent). Er ist der negative binäre Logarithmus des ersten nicht mehr toxischen Verdünnungsfaktors in einer Verdünnungsreihe mit dem Verdünnungsfaktor 2 (Krebs 2000). Die durch die höchsten pT-Werte ( $\mathrm{pT}_{\max }$-Werte) definierten Toxizitätsklassen werden mit römischen Zahlen gekennzeichnet.

Die mithilfe der pT-Wert-Methode ermittelten Toxizitätsklassen werden in Bezug auf die Handhabung von Baggergut den Handhabungskategorien „nicht“, „unbedenklich“, „kritisch“ und „gefährlich belastet" zugeordnet. Der angegebene Farbcode kennzeichnet die ermittelten Handhabungskategorien (Tab. 1).

Im Bereich der Küstenbundeswasserstraßen gelten geringfügig andere Randbedingungen.

\subsection{Verwendung, Verwertung und Beseitigung}

Ist eine Umlagerung nicht möglich, ist zu prüfen, ob das Material außerhalb der Bundeswasserstraßen verwendet oder verwertet werden kann. Hierbei sind das Wasserrecht (WHG) und die entsprechenden Landeswassergesetze zu beachten.

Bei der Verwertung von Baggergut an Land müssen gegebenenfalls konkurrierende Rechtsvorschriften zum Boden-, Natur-, Gewässer- und Immissionsschutz berücksichtigt werden. Nach dem BBodSchG stehen die Nachhaltigkeit und Sicherung der Bodenfunktion und die Vorsorge gegenüber schädlichen Bodenveränderungen im Vordergrund. Das Aufbringen von Baggergut unterliegt im Allgemeinen immer einer Einzelfallbetrachtung, wobei in der Praxis meist noch die Anforderungen an die Verwertung von mineralischen Abfällen (LAGA M20) angewandt werden. In einzelnen Bundesländern sind z.T. eigene Verwaltungsvorschriften vorhanden, die es einzuhalten gilt.

Wenn Baggergut aufgrund seiner Beschaffenheit weder einer Umlagerung noch einer Verwendung oder Verwertung zugeführt werden kann, dann wird es zur Beseitigung dem Abfallrecht unterworfen. Die Beseitigung hat auf abfallrechtlich zugelassenen Deponien zu erfolgen.

\subsection{Aktuelle Entwicklung am Rhein}

Eine Expertenarbeitsgruppe innerhalb der Internationalen Kommission zum Schutz des Rheins (IKSR) arbeitete eine Gesamtstrategie für ein Management von kontaminierten Sedimenten am Rheinstrom und den großen Nebenflüssen aus. Dieser Managementplan wurde als Empfehlung an die verantwortlichen Behörden für die Erfüllung des Rheinübereinkommens gemäß Artikel 3 zur „Verbesserung der Sedi-
Tab. 1 Ökotoxikologische Sedimentklassifizierung nach HABAB-WSV (BfG 2000), Toxizitätsklassen und Handhabungskategorien

\begin{tabular}{|c|c|c|c|c|c|c|}
\hline \multirow{2}{*}{$\begin{array}{l}\text { Höchste Verdün- } \\
\text { nungsstufe } \\
\text { Ohne Effekt }\end{array}$} & \multirow{2}{*}{$\begin{array}{l}\text { Verdünnungs- } \\
\text { faktor }\end{array}$} & \multirow{2}{*}{ pT-Wert } & \multicolumn{2}{|c|}{ Toxizitätsklassen } & \multicolumn{2}{|c|}{ Handhabungskategorien } \\
\hline & & & $\begin{array}{l}\text { 7-stufiges } \\
\text { System }\end{array}$ & Bezeichnung & $\begin{array}{l}\text { 4-stufige } \\
\text { Bewertung }\end{array}$ & Bezeichnung \\
\hline Originalprobe & $2^{0}$ & 0 & 0 & $\begin{array}{l}\text { Toxizität nicht } \\
\text { nachweisbar }\end{array}$ & 0 & Nicht belastet \\
\hline $1: 2$ & $2^{-1}$ & 1 & I & $\begin{array}{l}\text { Sehr gering } \\
\text { toxisch belastet }\end{array}$ & I & Unbedenklich \\
\hline $1: 4$ & $2^{-2}$ & 2 & II & $\begin{array}{l}\text { Gering toxisch } \\
\text { belastet }\end{array}$ & II & Belastet \\
\hline $1: 8$ & $2^{-3}$ & 3 & III & $\begin{array}{l}\text { Mäßig toxisch } \\
\text { belastet }\end{array}$ & III & Kritisch \\
\hline $1: 16$ & $2^{-4}$ & 4 & IV & $\begin{array}{l}\text { Erhöht toxisch } \\
\text { belastet }\end{array}$ & IV & Belastet \\
\hline $1: 32$ & $2^{-5}$ & 5 & V & $\begin{array}{l}\text { Hoch toxisch } \\
\text { belastet }\end{array}$ & V & Gefährlich \\
\hline$\leq(1: 64)$ & $\leq 2^{-6}$ & $\geq 6$ & VI & $\begin{array}{l}\text { Sehr hoch } \\
\text { toxisch belastet }\end{array}$ & VI & Belastet \\
\hline
\end{tabular}




\section{Entscheidungsfindung zur Ausweisung von Risikogebieten \\ - Konzept regelbasierte Bewertung -}

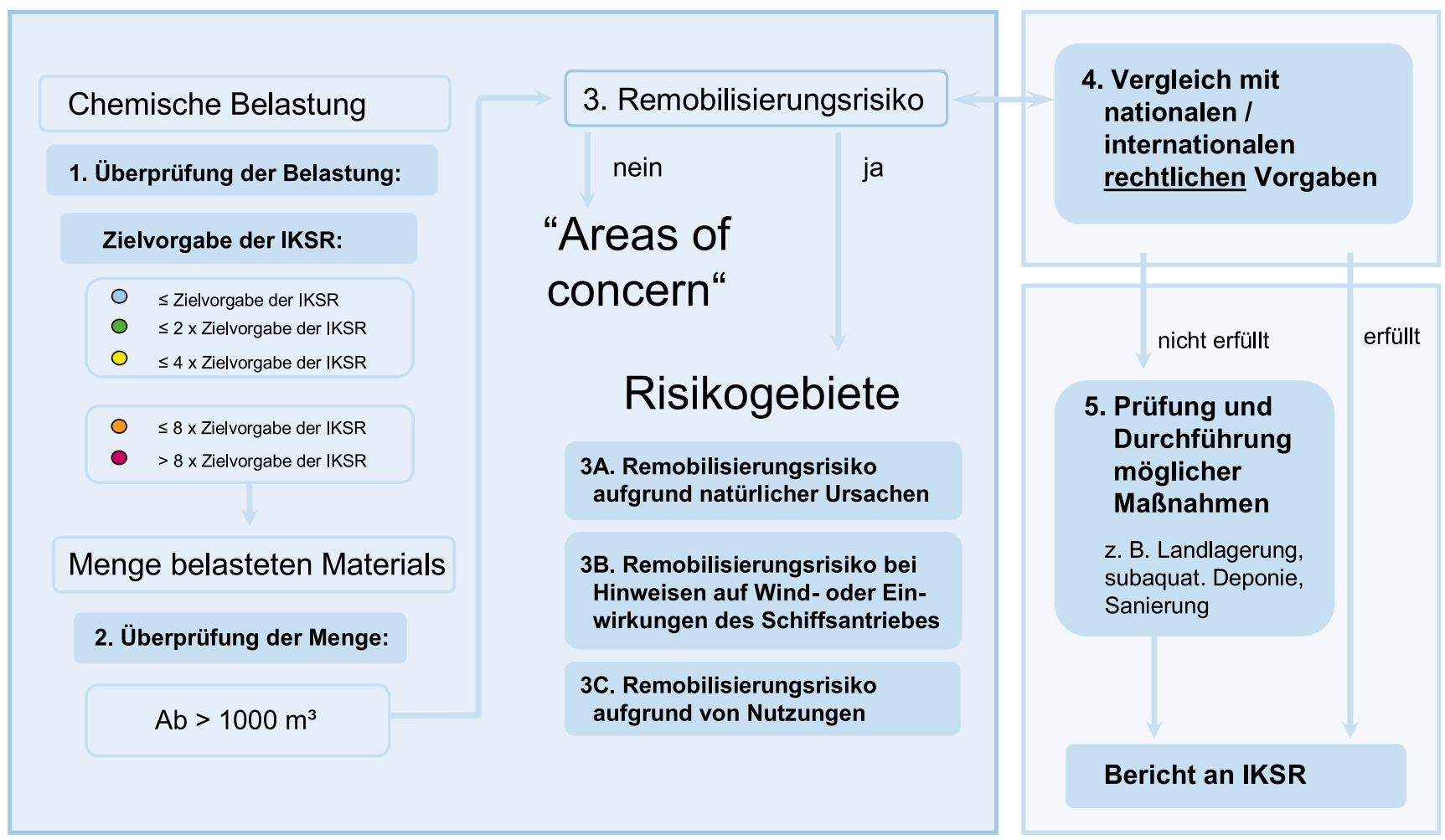

Abb. 1 Schema zur regelbasierten Entscheidungsfindung von Risikogebieten schadstoffbelasteter Sedimente

mentqualität für die schadlose Verbringung von Baggergut" sowie für die Aufstellung der Maßnahmenprogramme in die WRRL-Bearbeitungsgebiete weitergegeben. Die Erstellung des Managementplans umfasste zunächst die Bestandsaufnahme kontaminierter Sedimente unter Einbeziehung bisheriger relevanter Sedimentstudien. Dies beinhaltet die Erfassung von Menge und Qualität der mit Schadstoffen belasteten Sedimente, die Bewertung und Klassifizierung der Sedimente gemäß ihrem Gefährdungspotenzial sowie Vorschläge für einen ökonomisch und ökologisch vertretbaren Umgang mit den Sedimenten der verschiedenen Sedimentklassen (Abb. 1). Auch die nationalen Vorschriften sowie internationale Empfehlungen und Handlungsstrategien zum Sediment- und Baggergutmanagement, die bisher im Rheingebiet angewandt wurden, sind in dem Gesamtkonzept berücksichtigt. Darüber hinaus wurde eine Übersicht über die Risikogebiete einschließlich der Prioritäten der Behandlung erstellt (Abb. 2).

Für die Ausarbeitung des Sedimentmanagementplans wurden die Ergebnisse des IKSR-Forschungsprojektes „Untersuchungen zum Resuspensionsrisiko von Sedimentablagerungen in ausgewählten Staustufen des Rheingebie- tes“ aus den Jahren 2000-2003 zu Grunde gelegt (Witt et al. 2003). Die Untersuchungen kontaminierter Sedimentbereiche ergaben unter anderem einen Schwerpunkt in bestimmten Staustufen des Oberrheins, die durch eine historische, über viele Jahre andauernde Einleitung von Hexachlorbenzen (HCB) verunreinigt sind. Speziell Sedimentbereiche im Oberwasser der Staustufen Marckolsheim und Rhinau sind stark mit HCB belastet und stellen damit heute eine Quelle für eine Kontamination der Sedimente weiter flussabwärts dar (Tab. 2).

Tab. 2 Konzentrationen von Hexachlorbenzen in den Sedimenten der Oberrheinstaustufen (Untersuchungen aus dem Jahr 2000-2002)

\begin{tabular}{lcc}
\hline Stauhaltung & $\begin{array}{c}\text { Mittelwert } \\
\text { in } \boldsymbol{\mu g} / \mathbf{k g}\end{array}$ & $\begin{array}{c}\text { Maximaler Wert } \\
\text { in } \boldsymbol{\mu g} / \mathbf{k g}\end{array}$ \\
\hline Iffezheim & 158 & 910 \\
Gambsheim & 128 & 400 \\
Strasbourg & 223 & 2300 \\
Gerstheim & 135 & 1500 \\
Marckolsheim & 609 & 4100 \\
\hline
\end{tabular}


Abb. 2 Karte der Risikogebiete im Rheineinzugsgebiet mit Typisierung des Risikos

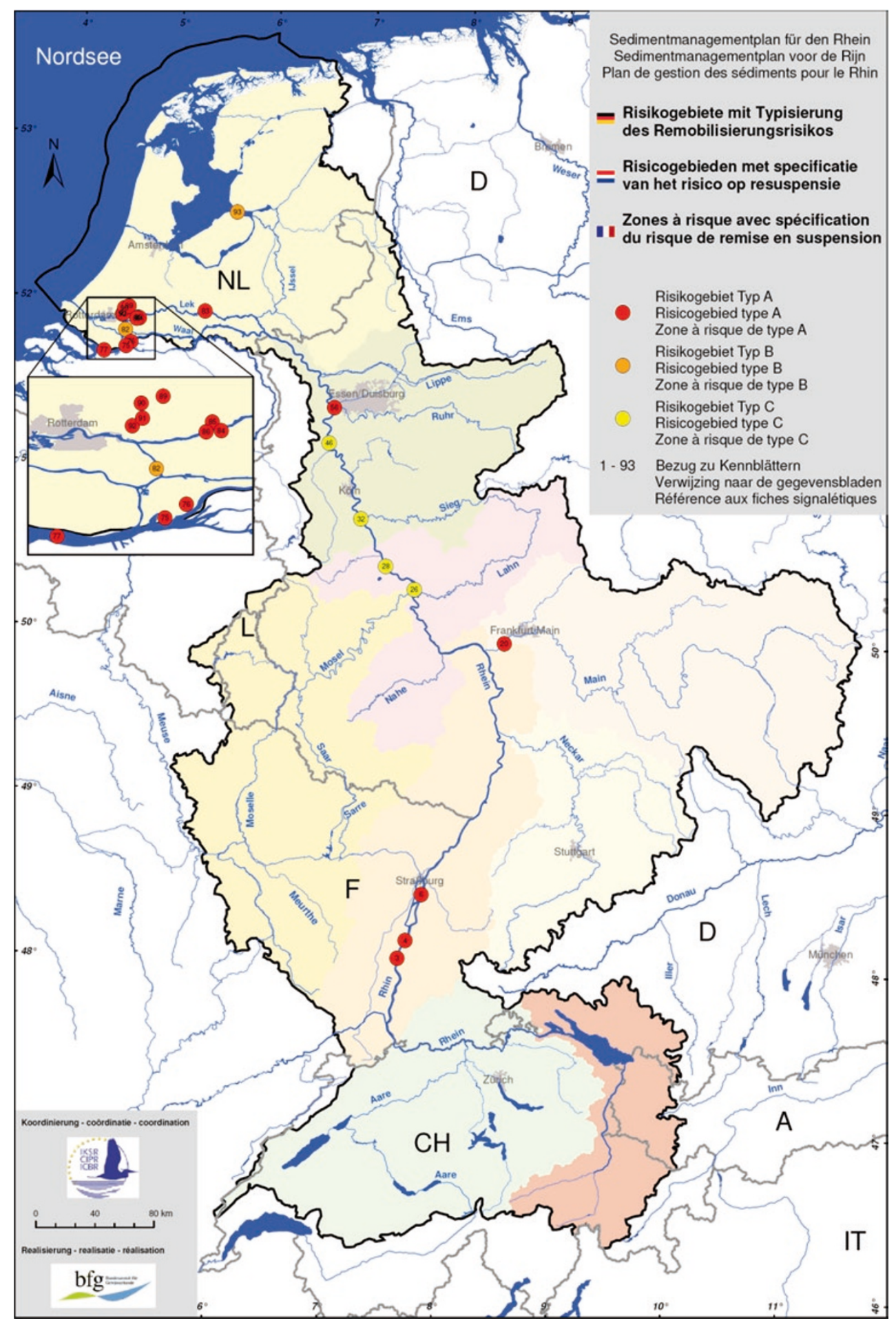

Das Konzept für den Sedimentmanagementplan Rhein basiert im Wesentlichen auf den Empfehlungen des europäischen Sedimentnetzwerkes SedNet. Das Konzept beinhaltet eine regelbasierte Bewertung mit folgenden drei Schritten:

- Identifizierung und Klassifizierung der relevanten Schadstoffe im Rheingebiet,
- Identifizierung und Klassifizierung der Gebiete mit relevanten verunreinigten Sedimentmengen und

- Identifizierung und Klassifizierung der Risikogebiete. Als relevante Schadstoffe im Rhein sind sechs Schwermetalle und organische Schadstoffe wie PAK, HCB und PCB benannt. Für den Grad der Verunreinigung wurde eine 5-stufige Klassifizierung auf der Basis der IKSR-Zielvor- 
gaben (Statusbericht Rhein 1993) gewählt. Das Remobilisierungsrisiko von verunreinigten Sedimenten wird anhand von Ergebnissen aus adäquaten Hochwasseruntersuchungen abgeschätzt oder durch Vergleich der kritischen Sohlschubspannung der Sedimente mit den Sohlschubspannungen, die bei einem Hochwasser der Jährlichkeit 10 auftreten können. Von den insgesamt untersuchten 93 Sedimentbereichen wurden 22 als Risikogebiete identifiziert und weitere 18 als Gebiete, die einer besonderen Aufmerksamkeit bedürfen (,,areas of concern“). Abbildung 2 gibt hierzu einen Überblick; sie ist auch als interaktive Karten unter http://www.iksr.org zu finden. Für die hochbelasteten Sedimentbereiche in den Stauhaltungen von Marckolsheim und Rhinau wird empfohlen, eine Prüfung mit dem Ziel der Sanierung vorzusehen, weil diese Bereiche heute eine Quelle für die HCB-Kontamination der Sedimente flussabwärts darstellen.

Eine erweiterte Zusammenfassung des IKSR-Sedimentmanagementplanes ist auf der Homepage der IKSR unter http://www.iksr.org/index.php?id=190\&tx_ttnews[tt_news]= 496\&tx_ttnews[backPid] $=58 \& \mathrm{cHash}=519 \mathrm{e} 9 \mathrm{~b} 560 \mathrm{c}$ zu finden.

\section{Zukünftige Arbeiten}

Aufgrund der geänderten rechtlichen Rahmenbedingungen aus derEU-WRRL, derFauna-Flora-Habitat-Richtlinie sowie dem Bundesnaturschutzgesetz und unter Berücksichtigung neuer Anforderungen aus internationalen Baggergutrichtlinien werden die Handlungsanweisungen HABAB und Gemeinsame Übergangsbestimmungen in einer gemeinsamen Handlungsanweisung zusammengeführt werden. Hierbei muss sichergestellt sein, dass sowohl das Handeln der Was- ser- und Schifffahrtsverwaltung in Übereinstimmung mit den Zielen der Flussgebietsgemeinschaften (z.B. FGG) erfolgt, andererseits die Handlungsziele der WSV durch die Flussgebietsgemeinschaften angemessen berücksichtigt werden. Da sich die qualitativen und quantitativen Aspekte des Baggergut- und Sedimentmanagements vielfach nicht voneinander trennen lassen, müssen in einer neuen Handlungsanweisung beide Aspekte entsprechende Wertung finden.

Im Rahmen der BMVBS-Klimainitiative KLIWAS (2009-2013) sind die klimatisch bedingten Veränderungen in Bezug auf die quantitativen und damit verbundenen qualitativen Aspekte des Sedimentmanagements Gegenstand von Untersuchungen sowohl für den Oberrhein als auch für die deutsche Elbe. Neben der Erweiterung der Prozess- und Systemkenntnisse soll die Entwicklung von Szenarien und Prognosen für das Sedimentmanagement stehen. Beides soll durch eine geeignete Kombination aus ein- und mehrdimensionalen Modellen zum Sediment- und Schadstofftransport umgesetzt und erweitert werden.

\section{Literatur}

BfG (2000) Handlungsanweisung für den Umgang mit Baggergut im Binnenland (HABAB-WSV), BfG-1251

BMVBS (2009) Gemeinsame Übergangsbestimmungen zum Umgang mit Baggergut in den Küstengewässern, WS14/5249.2/0

Krebs F (2000) Ökotoxikologische Bewertung von Baggergut aus Bundeswasserstraßen mit Hilfe der pT-Wert-Methode. Hydrol Wasserbewirtsch 44:301-307

Statusbericht Rhein (1993) Internationale Kommission zum Schutz des Rheins. Koblenz, September 1993. http://www.iksr.org/ index.php?id=134. Letzter Zugriff 29.9.2010

Witt O, Keller M, ten Hulscher D, Lehmann M, Westrich B (2003) Untersuchungen zum Resuspensionsrisiko belasteter Sedimentablagerungen im Rhein. Wasser 101:189-204 